\title{
Energy Scheduling in loE-Enabled Smart Grids Using Probabilistic Delayed Double Deep Q-Learning (P3DQL) Algorithm
}

This paper was downloaded from TechRxiv (https://www.techrxiv.org).

\section{LICENSE}

CC BY 4.0

SUBMISSION DATE / POSTED DATE

01-03-2022 / 03-03-2022

\section{CITATION}

Mohammadi Rouzbahani, Hossein; karimipour, Hadis; lei, lei (2022): Energy Scheduling in loE-Enabled Smart Grids Using Probabilistic Delayed Double Deep Q-Learning (P3DQL) Algorithm. TechRxiv. Preprint. https://doi.org/10.36227/techrxiv.19282859.v1

$\mathrm{DOI}$ 


\title{
Energy Scheduling in IoE-Enabled Smart Grids Using Probabilistic Delayed Double Deep Q-Learning (P3DQL) Algorithm
}

\author{
Hossein Mohammadi Rouzbahani, Hadis Karimipour, Lei Lei
}

\begin{abstract}
Decentralization and high penetration of smart devices in IoE-enabled smart grids face the power system with complex scheduling problems. Engaging with big data produced by the interconnected infrastructures, besides the high dimensional and uncertain environment, make traditional methods incapable of addressing these problems since exact modeling of the environment under uncertainties is impracticable. Also, learning-based methods suffer from excessive complexity and the curse of dimensionality. This research proposes a Probabilistic Delayed Double Deep QLearning (P3DQL) which is a combination of the tuned version of Double Deep Q-Learning (DDQL) and Delayed Q-Learning (DQL). The planned algorithm makes a trade-off between overestimation and underestimation biases guaranteeing efficiency regarding sample complexity and learning proficiency by applying a delay in updating the rule. Finally, the proposed algorithm is tested on real-world data from Pecan Street Inc., assessing the performance of the P3DQL regarding peak clipping, decreasing Peak to Average Power Ratio (PAPR), and cost reduction. The results indicate the superiority of the developed algorithm over other utilized methods by $28.2 \%$ peak clipping, $12.9 \%$ PAPR decrease, and $29.4 \%$ cost reduction.
\end{abstract}

Index Terms-Energy Scheduling, Smart Grid, Deep Reinforcement Learning, Double Q Learning, PAC Learning, Internet of Energy

\section{INTRODUCTION}

$\mathrm{O}$ ne of the key challenges in power systems is harmonizing demand and generation in an efficient manner. Although Economic Dispatch (ED) and Unit Commitment (UC) are two well-investigated problems aiming to deal with the abovementioned concern, high penetration of distributed resources, Electric Vehicles (EVs), Advanced Metering Infrastructures (AMIs), and telecommunication networks open new challenges and perspectives in addressing scheduling problems [1]. Playing an active role by consumers in Demand Response (DR) programs makes utilities capable of orchestrating the balance between demand and supply sides, especially during peak hours, to prevent running expensive power plants [2].

Conventional methods fail to solve new scheduling problems in smart grids due to complex non-linear environments in addition to their incapability of processing big data generated by different components of the smart grids. Likewise, heuristics and mathematical algorithms rely on explicit environment models and precise forecasts of various

Hossein Mohammadi Rouzbahani, Hadis Karimipour, are with University of Calgary, Calgary, AB, T2N 1N4, Canada (e-mail: hossein.mohammadirou@ucalgary.ca; hadis.karimipour@ucalgary.ca). Lei Lei is with University of Guelph, Guelph, ON, N1G 2W1, Canada (e-mail: leil@uoguelph.ca). types of uncertainties, including technical, economic, and weather uncertainties, that cannot be fulfilled in real-world problems. Even supervised learning methods that are less subordinate to accurate forecasting data and model uncertainties still suffer from limited approximation capability and slow convergence in a large-scale dynamic and decentralized environment that generates big data with considerable nonlinearity [3]. Since scheduling in the new generation of the smart grid is a decision-making NP-hard problem, Reinforcement Learning (RL) is a well-suited algorithm to solve this optimization problem by taking advantage of learning optimal behavior through making a trade-off between exploration and exploitation [4].

Artificial intelligence 2 (AI 2.0) and Internet of Energy (IoE) are two advanced concepts that are capable of tackling scheduling problems in fully interconnected large-scale networks with dynamic and uncertain environment models. While an IoE framework collects data from all energy kits providing intelligent real-time monitoring and dynamic control, AI 2.0 combines data-driven and experience-based engines by integrating natural and artificial intelligence in order to make the decision [5]. RL and Deep Reinforcement Learning (DRL) which are two well-known subcategories of AI 2.0, demonstrate outstanding performance that exceeds human intelligence. In March 2016, the world Go champion was defeated by the AlphaGo algorithm which had been developed by Google DeepMind based on DRL [6].

Subsequently, a large volume of research has been conducted to solve scheduling optimization problems in the new generation of smart grids, where all energy components are fully interconnected, collecting and sharing big data over an IoE framework to be processed by AI 2.0 family algorithms. A model-free RL algorithm has been proposed in [7], guarantying data confidentiality and consumers' privacy. In [8], a multi-agent optimizing scheme has been developed based on RL for solving routing and scheduling problems. The authors in [9], suggested a data-driven DRL-based scheduling algorithm for microgrid energy optimization. Remani et al. [10] presented an RL-based solution in order to minimize electricity costs for the end-users considering Photovoltaic (PV) generation uncertainty. In [11], a model-free Q-Learning framework has been developed to schedule operational time of home appliances considering a roof top PV system and an energy storage unit. The authors in [12] developed a QLearning algorithm to schedule multiple appliances in a smart home, preserving customer welfare and preferences.

Many challenges are associated with RL, including the curse of dimensionality, lack of scalability, poor 
generalization, and limited non-linear representation capability that make this method disqualified from solving scheduling problems in a real-world smart grid environment [3], [13]. DRL algorithms have recently accomplished extraordinary breakthroughs exploiting Deep Neural Network (DNN) strengths, involving the ability to handle unstructured data, no need for feature engineering, and non-linear representation capability, to name a few.

Deep Q-Learning (DQL) has been utilized in [14] and [15], to specify the optimal scheduling policy to schedule the workflow of heterogeneous virtual machines, respectively. David et al. [16] proposed a DQN-based solution learning optimal policy for the operation of energy entities in a microgrid aiming to minimize cost. In [17], a scheduling problem aiming to address supply-demand mismatch in microgrid energy trading has been solved using DRL. A model free DQL approach has been introduced in [18] to determine an optimal strategy for real-time scheduling of Electric Vehicle (EV) charging. The authors in [12] compared DQL and Deep Policy Gradient (DPG) methods, where expectedly, DPG demonstrated a better performance. The superiority of DPG over DQL in this optimization problem originated from overestimation bias in Q-Learning. As a solution, Double DQL (DDQL) reduces the positive bias of QLearning that has not been investigated in [19]. Chung et al. [20] proposed a DPG algorithm for scheduling the operation of home appliances preserving their privacy. Two versions of DPG, including Centralized Deterministic DPG (C-DDPG) and Distributed Deterministic DPG (D-DDPG), have been developed making a comparison among results with a stochastic weight averaging algorithm. As results show, sample efficiency and usability are still two key concerns in the proposed methods. Moreover, the difference in reward of four reported buildings reveals the model suffers from high variance. It is worth mentioning that policy gradient methods face the risk of trapping in a local optimal; also, a lot of training time is required to reach the global result.

Neither previously mentioned studies nor other related investigations in the literature tried to eliminate biases of DDQL (e.g., overestimation and underestimation concerns) in order to take advantage of the simplicity and stability of DQL. Likewise, learning efficiency and space and sample complexity that have not been well investigated in the previous works as mentioned earlier. While these characteristics incredibly affect time-efficient and costeffective solutions in discrete action spaces and problems with continuous and large action spaces.

The main contributions of this paper are summarized as follows.

1. An alternative version of the DDQL algorithm is proposed to make a trade-off between positive and negative bias, resulting in the overestimation and underestimation biases cutback.

2. The developed algorithm is modeled as a Probably Approximately Correct in Markov Decision Processes (PACMDP), enhancing learning efficiency and reducing sample complexity making the algorithm capable of handling problems with large action space.

3. A Probabilistic Delayed Double Deep Q-Learning (P3DQL) algorithm is developed and utilized for a scheduling problem in smart grids for the first time.

4. An optimal multi-layer scheduling mathematical model is proposed which comprehensively covers from a single Nano Area (NA) to a Neighborhoods (NH) and a Wide Area Network (WAN), including share storage units, PVs, and considering different tariff types and customer preferences. Subsequently, the problem is formulated as a Markov decision process (MDP) and solved by the P3DQL algorithm.

The remaining of this paper is structured as follows. Sections 2 and 3 present the system mathematical and MDP model of the problem. The proposed P3DQL algorithm is introduced in section 4. Results are provided in section 5 . Finally, a brief conclusion is presented in section 6 .

\section{MAthematical Formulation OF The Proposed MULTI-LAYER MODEL}

The proposed model is an autonomous and dynamic framework enabling optimal scheduling at different levels, including NAs, NHs, and WANs. The model's layout is shown in Figure 1, where all components are interconnected in different layers taking advantage of a bi-directional communication enabled by the IoE. A micro area is a smart home that consists of various appliances as well as a Private PV (PPV) cell and/or EV(s). Consequently, two or more houses can form a neighborhood that includes an Electricity Storage System (ESS), Shared PV (SPV) modules, and an Electric Vehicle Charging-station (EVC). Finally, a wide network area can be composed of a couple of neighborhoods.

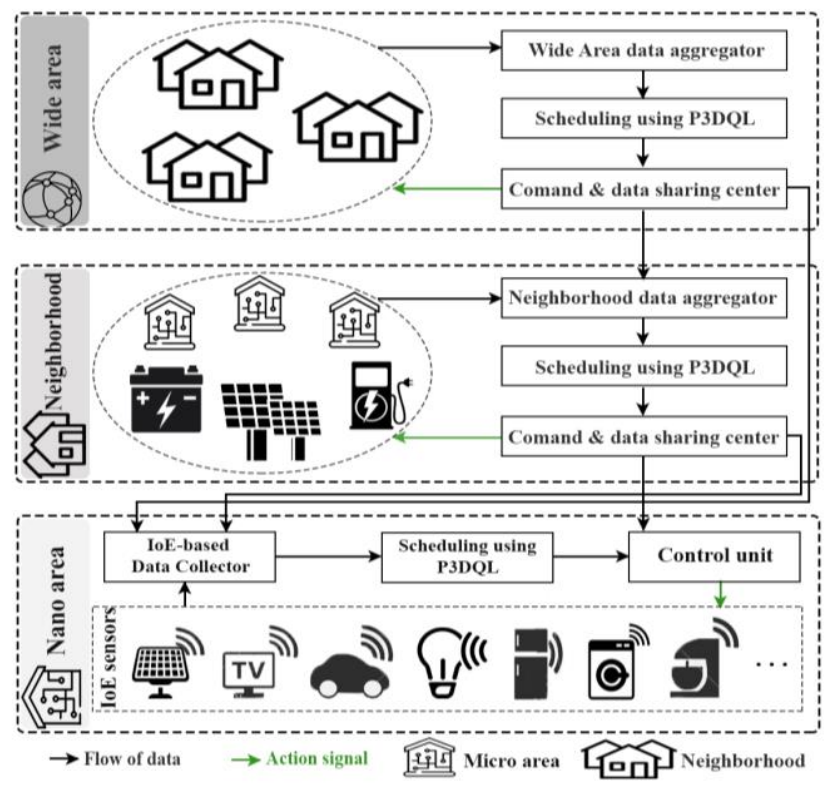

Figure 1. The layout of the proposed model

The designed model has a highly dynamic environment where each NA is able to participate in one or more NHs at the same time or even in different time slots based on their contracts for electricity trading. Likewise, each ESS, SPV, and 
EVC cab be exploited by one or more NHs. Equations (1) and (2) define the matrix of WAN agents $A_{M \times 1}^{W A N}$ in a specific time slot, where $H \in \mathbb{N}^{N \times 1}$ denotes a set NAs, $R \in \mathbb{N}^{3 \times 1}$ stands for the matrix of shared units demonstrating the type and capacity of the module, also $\Delta \in \mathbb{N}^{M \times N}$ and $\Psi \in \mathbb{N}^{M \times 3}$ are activation matrixes for micro areas and shared units, respectively. Therefore, sequentially $\delta_{i j}$ and $d_{i j}$ demonstrate the participating status of micro area and energy unit $j$ in $\mathrm{NH}$ number $i$, respectively.

$[A]_{M \times 1}=\left([\Delta]_{M \times N} \times[H]_{N \times 1}\right)+\left([\Psi]_{M \times 3} \times[R]_{3 \times 1}\right)$

$\left[\begin{array}{c}a_{1} \\ \vdots \\ a_{M}\end{array}\right]=\left[\begin{array}{ccc}\delta_{11} & \cdots & \delta_{1 m} \\ \vdots & \ddots & \vdots \\ \delta_{m 1} & \cdots & \delta_{m n}\end{array}\right] \times\left[\begin{array}{c}h_{1} \\ \vdots \\ h_{n}\end{array}\right]+\left[\begin{array}{lll}\psi_{11} & \psi_{12} & \psi_{13} \\ \psi_{12} & \psi_{22} & \psi_{23} \\ \psi_{31} & \psi_{32} & \psi_{33}\end{array}\right] \times\left[\begin{array}{c}E S S \\ S P V \\ E V C\end{array}\right]$

The model aims to reduce the peak to average ratio and load variance while minimizing the cost at all levels. Let $R P_{t}=\Omega_{t} \Phi_{t} \Pi_{t} \lambda$ represents the retail price in time slot $t$ under the given incentive factor $\Omega$, penalty factor $\Phi$, and price of electricity $\lambda$. Additionally, contract coefficient $\Pi$ is defined based on the terms and conditions of energy trading between units during a specific period of time $(\Pi=1$ means there is no contract). Equations (3) and (4) describe the incentive and penalty factors, where $\Psi_{t}$ is the total consumption in time frame $t$, also lower and higher pricing threshold of net load are defined by $\theta_{1}$ and $\theta_{2}$, respectively. It should be noted that incentive and penalty factors and both thresholds are estimated to calculate the real-time price considering the contract coefficient.

$\Omega_{t}= \begin{cases}\bar{\Omega} & 0<\bar{\Omega}<1 \stackrel{\text { if }}{\Rightarrow} t \in \text { off }- \text { peak } \\ \underline{\Omega} & \underline{\Omega}>1 \stackrel{\text { if }}{\Rightarrow} t \in \text { peak hours } \\ 1 & \underline{\Omega}=1 \stackrel{\text { if }}{\Rightarrow} t \in \text { mid }- \text { load hours }\end{cases}$

$\Phi_{t}= \begin{cases}\bar{\Phi} & 0<\bar{\Phi}<1 \stackrel{\text { if }}{\Rightarrow} \Psi_{t}<\theta_{1} \\ 1 & \underline{\Omega}=1 \stackrel{\text { if }}{\Rightarrow} \theta_{1} \leq \Psi_{t} \leq \theta_{2} \\ \underline{\Phi} & \bar{\Phi}>1 \stackrel{\text { if }}{\Rightarrow} \Psi_{t}>\theta_{2}\end{cases}$

\section{A. Scheduling problem formulation in NA}

Three types of costs are specified and objective functions in each NA, including monetary cost $f_{11}$, instruments degradation cost $f_{12}$, and the consumer discomfort cost $f_{13}$. Consequently, the formulation of the primary objective functions of the NA level is as follows.

$\min \sum_{i \in \omega} F_{1 i}$

$F_{1}=\left(f_{11}, f_{12}, f_{13}\right)$

$f_{11}=\sum_{t=1}^{T}\left(\sum_{j=0}^{\omega}\left(R P_{t}\right) \Psi_{j t}+{ }_{y}^{N A} \xi_{t}{ }_{y}^{N A} l_{t}\left(\Gamma_{t}^{i}-\Gamma_{t}^{e}\right)\right)$

$f_{12}=\sum_{j=0}^{\omega} D_{j}(\check{T} ; \vartheta, k)=\sum_{j=0}^{\omega} \frac{\kappa_{j}}{\vartheta_{j}}\left(\frac{\breve{T}_{j}}{\vartheta_{j}}\right)^{\kappa_{j}-1} \cdot e^{-\breve{T}_{\vartheta_{j}} k}$

$f_{13}=\sum_{j=0}^{\omega} \eta_{j}\left(\sum_{t=1}^{T} \zeta_{t}^{d}-\zeta_{t}^{a}\right)+\sum_{t=1}^{T}\left|{ }_{a}^{o} c_{t}-{ }_{d}^{o} c_{t}\right|$

Cost minimization problem at the NA level must also fulfill the following constraints:
$\sum_{t=1}^{T} \sum_{j=1}^{\omega} \Psi_{j t}=L_{\text {total }}, \forall t \in \mathbb{N}, \forall j \in \mathbb{N}$

$0 \leq\left|\Gamma_{t}\right| \leq L_{\text {total }}, \forall t \in \mathbb{N}, \Gamma_{t} \in \mathbb{R}$

$D_{j}(\breve{T} ; \vartheta, k)>0, \forall \breve{T} \in \mathbb{N}, \forall \vartheta \in \mathbb{R}^{+}, \forall \kappa \in \mathbb{R}^{+}$

$\xi_{t}>1, \eta_{j}>1, \forall t=[1: T] \in \mathbb{N}, \forall j=[1: \omega] \in \mathbb{N}$

$\zeta_{t}^{d} \in\{0,1\}, \zeta_{t}^{a} \in\{0,1\}, \zeta_{t}^{d} \neq \zeta_{t}^{a}, \forall t=[1: T] \in \mathbb{N}$

The first objective $f_{11}$ aims to minimize the bill considering; the imported power $\Gamma_{t}^{b}$, the exported power $\Gamma_{t}^{b}$, and the transmission fee coefficient ${ }^{N A} \xi_{t}$ and loss coefficient ${ }^{N A} l_{t}$ at each time frame $t$ in $y^{t h} \mathrm{NA}$, where $T$ and $\omega$ are the total number of time slots and appliances, respectively. Besides, ${ }^{N A} L_{\text {total }}$ is the total power consumption of NA number $y$. The second objective focuses on the degradation cost of electrical devices based on the Weibull distribution function [21]. Accordingly, $\breve{T}_{j}$ defines the cumulative time of operating $j^{\text {th }}$ appliance, $\vartheta$ is the Weibull scale parameter, and $\kappa$ stands for the Weibull shape parameter. The third objective is interested in discomfort minimization, taking operational delay and desired indoor temperature into account at the same time. Also, $\eta$ indicates the importance of on-time operation for each appliance, considering the difference between the desired operational status $\zeta_{t}^{d}$ and actual status $\zeta_{t}^{a}$.

\section{B. Scheduling problem formulation in $\mathrm{NH}$}

The mathematical scheduling model of an $\mathrm{NH}$ is presented comprising multiple NAs $h_{i}$ and shared units $R^{j} \in$ $\mathbb{N}^{3 \times 1}$ Where $i$ and $j$ are the IDs of participated NAs and set of RES in the given NH. Since all NAs are considered to be optimized in the prior level, they are inspected as black boxes with hidden internal mechanisms that just swap electricity with the $\mathrm{NH}$. The first objective $f_{21}$ intends to minimize transaction costs among all participated units, also other $\mathrm{NHs}$ and WANs. Minimizing ESS loss $P_{E S S}^{\text {loss }}$, and PV loss $P_{P V}^{\text {loss }}$ [22] are the key goals of the second objective, $f_{22}$. It should be pointed out that ESSs involve both fixed and mobile electricity storage (EV). The third objective $f_{23}$ minimizes EVC cost considering various potential generation sources, while the fourth objective investigates the maintenance and degradation costs $f_{24}$. Ultimately, the scheduling problem at the NH level is formulated as follows.

$\min \left(\sum_{i \in h^{*}} F_{2 i}+\sum_{l \in R^{j}} F_{2 i}\right)$

$F_{2}=\left(f_{21}, f_{22}, f_{23}, f_{24}\right)$

$f_{21}=\sum_{t=1}^{T}\left(\sum_{w \in \Phi} \sum_{k \in \Phi}{ }_{z}^{N H} l_{t}^{w k} \cdot P_{t}^{w k}\right)+{ }_{y}^{N H} \xi_{t}{ }_{y}^{N H} l_{t} \Gamma_{t}^{*}$

$f_{22}=\sum_{t=1}^{T}\left(\lambda_{\text {bat }}{ }_{t}^{-\alpha}-1\right) P_{\text {bat }}{ }_{t}^{\alpha}+\lambda_{\text {conv }} \hat{A}_{p v} I_{t} \tau_{t}$

$f_{23}=\sum_{t=1}^{T} \beta_{1} P_{E V C_{t}}{ }^{2}+\beta_{2} P_{E V C_{t}}+\beta_{3}$

$f_{24}=\sum_{r \in R} D_{r}(\breve{T} ; \vartheta, k)=\sum_{r \in R} \frac{\kappa_{r}}{\vartheta_{r}}\left(\frac{\breve{T}_{r}}{\vartheta_{r}}\right)^{\kappa_{r}-1} \cdot e^{-\left(\frac{\breve{T}_{r}}{\vartheta_{r}}\right)^{k}}$

Equations (21) to (28) illustrate the constraints that must be satisfied in the scheduling problem.

$\Phi \in\left\{h_{1}, \cdots, h_{n}\right\} \cup\left\{R E S_{i}, P V_{i}, E V C_{i}\right\}, \forall n \in \mathbb{N},, \forall j \in \mathbb{N}$ 
$0 \leq{ }_{z}^{N H} l_{t}^{w k}<1,\left\{{ }_{y}^{N H} \xi_{t},{ }_{y}^{N H} l_{t}\right\} \geq 1, \Gamma_{t}^{*} \geq 0,, \forall t=[1: T]$

$\alpha \in\{0,1\}, \lambda_{\text {bat }_{t}}^{-\alpha} \leq 1, \lambda_{\text {conv }_{t}} \leq 1, \forall t=[1: T] \in \mathbb{N}$

$\operatorname{SOC}_{E S S_{t+1}} \leq \operatorname{SOC}_{E S S_{t}}+\left(\lambda_{\text {bat }}{ }^{-\alpha}-1\right) P_{\text {bat }}{ }_{t}, \forall t=[1: T] \in \mathbb{N}$

$S O C_{E S S_{\text {min }}} \leq S O C_{E S S_{t}} \leq S O C_{E S S_{\text {max }}}$

$\tau_{t}=1-0.005\left({ }_{\text {outside }}^{o} c_{t}-25\right), t=[1: T] \in \mathbb{N}$

$\beta_{j}>0, P_{E V C_{t}} \geq 0, j \in\{1,2,3\}, t=[1: T] \in \mathbb{N}$

$D_{r}(\breve{T} ; \vartheta, k)>0, \forall \breve{T} \in \mathbb{N}, \forall \vartheta \in \mathbb{R}^{+}, \forall \kappa \in \mathbb{R}^{+}, r \in R$

Where $\Phi$ is a set of NAs joined in the NH, ${ }_{z}^{N H} l_{t}^{w k}$ stands for power loss of swapping electricity from $w$ to $k, P_{t}^{w k}$ demonstrates the quantity of transferred power between entities, ${ }^{N H} \xi_{t},{ }_{y}^{N H} l_{t}$, and $\Gamma_{t}^{*}$ are transmission fee coefficient, loss coefficient, and exchanged power from/to the $\mathrm{NH}$, respectively. Furthermore, $\lambda_{\text {bat }}$ denotes the efficiency of the ESS, $\lambda_{\text {conv }}{ }_{t}$ represents the converting efficiency of the PV, $\alpha$ symbolizes the charging $(\alpha=1)$ and discharging $(\alpha=-1)$ status, $S O C_{E S S_{t}}$ defines the state of charge of the storage at time slot $t$. Finally, outside ${ }^{o} c$ indicates the outside temperature in Celsius, $P_{E V C_{t}}$ stands for the total power of the charging station at time slot $\mathrm{t}$, and $D_{r}$ denotes the degradation of each $r \in\{R E S, P V, E V C\}$.

\section{Scheduling problem formulation in WAN}

From the standpoint of the WAN level, each NA and NH endeavor to optimize the different types of costs and losses considering their subdivision entities, which leads to WAN scheduling problems focused on minimizing electricity trading costs among WANs as given in Equation (29).

$\min \sum_{t=0}^{T}\left(\sum_{w \in \Upsilon} \sum_{k \in \Upsilon}{ }^{W A N} \xi_{t} \cdot{ }^{W A N} l_{t}^{w k} . P_{t}^{w k}\right)$

Where $\Upsilon$ is the set of contributed WANs in the scheduling optimization plan.

\section{MDP FORMULATION OF THE PROPOSED MULTI- LAYER MODEL}

The MDP formulation of the multi-layer power system model is presented in this section. The proposed formulation is configured in three different stages whereby each level is considered as a single energy entity in the next level. Initially, five major controllable appliances, including Air Conditioner (AC), Washing Machine (WM), Dryer (DY), Dishwasher (DW), and EV, are deemed in a smart house as a NA. Equation (30) illustrates the state space of a NA.

$s^{N A}{ }_{t}=\left\{l^{A C}{ }_{t}, l^{W M}{ }_{t}, l^{D Y}{ }_{t}, l^{D W}{ }_{t}, l^{E V}{ }_{t}, E^{P V}{ }_{t}, R P_{t}\right\}$

Where $l^{j}{ }_{t}$ denotes the power consumption of the appliance $j$ during time slot $t, E^{P V}{ }_{t}$ represents the state of the $\mathrm{PV}$, including internal dispensing or exporting to the upper level, and $R P_{t}$ indicates the retail price at $t$. From an NH point of view , ${ }_{i}^{N A} E_{t}$ is the state of $i^{\text {th }}$ NA which defines whether the NA exports or imports electricity during $t$. Likewise, states of ESS, SPV, and EVC are defined as ${ }_{i}^{E S S} E_{t},{ }_{i}{ }_{i} E_{t}$, and ${ }_{i}^{E V C} E_{t}$, respectively. Finally, from the WAN standpoint, the state space contains the energy status of each $\mathrm{NH}^{\mathrm{NH}}{ }_{i} E_{t}$, besides the swapping price between $i^{\text {th }}$ and $j^{\text {th }} \mathrm{NH}{ }_{i j}^{W} R P_{t}$. Equations (31) and (32) illustrate the state space of NHs and WANs, respectively.

$s^{N H}{ }_{t}=\left\{{ }_{1}^{N A} E_{t}, \ldots,{ }_{i}^{N A} E_{t},{ }_{i}^{E S S} E_{t},{ }_{i}^{S P V} E_{t},{ }_{i}{ }_{i} E_{t},{ }_{i j}^{N} R P_{t}\right\}$

$s^{W A N}{ }_{t}=\left\{{ }_{1}^{N H} E_{t}, \ldots,{ }_{i}^{N H} E_{t},{ }_{i j} R P_{t}\right\}$

The optimal action of each entity depends on the dedicated agent to maximize its reward. Equation (33) defines the action space of non-adjustable appliances, including WM, DY, and DW.

$A^{a p p}=\{$ On, Off, Hold $\}, \forall a p p \in\{W M, D Y, D W\}$

EVs are equipped with Grid-to-Vehicle (G2V) and Vehicle-to-Grid (V2G) technologies. Correspondingly, the action space of EVs is as follows.

$A^{E V}=\{G 2 V, V 2 G$, Disconect $\}$

The action space of $\mathrm{AC}$ is discretized into $2 n+1$ levels where $n$ is the temperature degrees of freedom given by the user. The agent is authorized to decrease or increase the temperature by $n$ degrees, one degree at a time, as Equation (35) shows. $T^{N A}$ denotes the current temperature, and ${ }_{-}^{+} x T^{N A}$ indicate $x$ degree of temperature change. The same logic applies to the ESS and EVC as indicated in Equations (36) and (37) where $E^{E S S}$ is the energy level of the shared unit, and $q$ defines the number of permitted changes in the SOC once at a time, $\Gamma^{E V C}$ denotes the price of supplied electricity by EVC, and $+r \Gamma^{E V C}$ stands for increasing price by $r$ unit of currency.

$A^{A C}=\left\{-n T^{N A}, \ldots,-1 T^{N A}, T^{N A},+1 T^{N A}, \ldots,+n T^{N A}\right\}$

$A^{E S S}=\left\{-q E^{E S S}, \ldots,-1 E^{E S S}, E^{E S S},+1 E^{E S S}, \ldots,+q E^{E S S}\right\}$

$A^{E V C}=\left\{\Gamma^{E V C},+1 \Gamma^{E V C}, \ldots,+r \Gamma^{E V C}\right.$, off $\}$

Both private and shared PV agents follow the same action space, Equation (35) explains, where the system is capable of switching the terminus consumption from internal to external (IE) or from external to internal (EI) units. Furthermore, the PV will be detached once output power drops under the economic power threshold. The action space of the rest of the entities, including the entire NA, NH, and WAN, also follow the same method.

$A^{Z}=\{I E, E I, O f f\}, \forall Z \in\{P V, S P V, N A, N H, W A N\}$

The key objective of the problem is to find the optimal policy $\pi\left(a_{t} \mid s_{t}\right)$, maximizing the total expected reward as follows.

$\max _{\pi} J(\pi)=\mathrm{E}_{\tau \sim \pi}\left[\sum_{t=o}^{T-1} R_{t}^{\text {total }} \cdot \gamma^{t}\right]$

Where $\tau=\left\{s_{0}, a_{0}, s_{1}, \ldots, a_{T-1}, s_{T}\right\}$ denotes the agent's trajectory, $\mathrm{E}_{\tau \sim \pi}[$.$] represents the expected value over \tau$, the probability of transition from $s_{t+1}$ to $s_{t}$ by action $a_{t}$ defines by the probability transition function $P\left(s_{t+1} \mid s_{t}, a_{t}\right), \gamma \in(0,1]$ is the discount factor indicating the importance of future rewards. Moreover, $R_{t}^{\text {total }}$ indicates the overall reward, 
defined independently for each level, as shown in Equations (40) to (42).

$R_{N A}^{\text {total }}=\sum_{t=0}^{T} \sum_{i \in D} r_{i_{t}}, D \in\{a p p, E V, A C, P V\}$

$R_{N H}^{\text {total }}=\sum_{t=0}^{T}\left(\sum_{i \in N A} r_{i_{t}}+\sum_{j \in G} r_{j_{t}}\right), G \in\{E S S, S P V, E V C\}$

$R_{N H}^{\text {total }}=\sum_{t=0}^{T} \sum_{i \in N H} r_{i_{t}}$

Ultimately, this research presents a comprehensive reward function that applies to all entities based on the feasible conditions indicated in Equation (40).

$$
r_{t}= \begin{cases}-\left(R P_{t} \Psi_{t}+\sum_{i \in \mho} \varphi_{i}\left|\varpi_{i}^{a}-\varpi_{i}^{d}\right|\right) & \varpi_{i}^{a} \neq \varpi_{i}^{d} \\ -R P_{t} \Psi_{t} & \varpi_{i}^{a}=\varpi_{i}^{d}\end{cases}
$$

Where $\mho$ is a set of criteria including cost, delay, loss, degradation, temperature, and monetary profit, $\varpi_{i}^{a}$ and $\varpi_{i}^{d}$ denote the actual and preferred quantity of criteria $i$, respectively, $\varphi_{i}$ represent the penalty factor of violating $i^{t h}$ criteria.

\section{The PROPOSEd ProbabILISTIC DELAYED DOUBLE DEEP Q-LEARNING (P3DQL) ALGORITHM}

Different stages of the P3DQL algorithm development are defined in this section. The first step is tuning the early version of DDQL, making a trade-off between overestimation and underestimation biases. The second stage aims to enhance sample complexity and learning proficiency by applying a delay in updating rules considering the nature of the problem guaranteeing efficiency. Finally, combining applied methods in the previous stages forms a Probably Approximately Correct (PAC) algorithm with an adjustable trade-off between positive and negative biases.

Primarily the transition from Q-Learning to the early version of DDQL is investigated to illustrate the significant concerns that require to be addressed by P3DQL. The classic Q-Learning relies on a look-up table taking the superior action at each state while storing the values of the state-value Qfunction. Unfortunately, besides the sluggish process, the quantity of required memory have made the Q-Learning algorithms worthless for real-world problems. DNN plays a role as a function approximator in DQL, where the inputs are the states, and the Q-values are calculated as the outputs, focusing on minimizing the loss function as shown in Equation (44).

$L\left(\theta_{t}\right)=\mathrm{E}_{\mu}\left[\left(Q\left(s, a ; \theta_{t}\right)-r_{t+1}-\gamma \max _{a} Q\left(s^{\prime}, a ; \theta_{t}\right)\right)^{2}\right]$

Where $Q(s, a)$ represents the value of action $a_{t}$ in state $s_{t}$, $\mu$ is the experience buffer containing, and $r$ denotes the reward. Also, the update of Q-Learning is as follows.

$Q_{t+1}(s, a) \leftarrow Q_{t}(s, a)+\alpha_{t}(s, a) \cdot \widehat{T D}_{t+1}(s, a)$

$\widehat{T D}_{t+1}(s, a)=r(s, a)+\gamma \max _{a^{\prime}} Q\left(s^{\prime}, a^{\prime}\right)-Q_{t}(s, a)$

Where $s^{\prime}$ is the next state by the probability of transferring from state $s$ with action $a, P\left(s, a, s^{\prime}\right) \rightarrow[0,1], \alpha_{t}$ denotes the learning rate that controls the velocity of adaptivity to randomness in the rewards and transitions.

Q-Learning is stricken with a significant positive bias originating from applying the $\max$ operator $\max _{a} Q\left(s^{\prime}, a ; \theta_{t}\right)$ in the update rule. Correspondingly, DDQL was introduced by Van-Hasselt [16], approximating the Q-values using two independent estimators to update $Q^{A}$ and $Q^{B}$ follows.

$\left\{\begin{array}{l}Q^{A}(s, a) \leftarrow Q^{A}(s, a)+\alpha\left(r+\gamma\left(Q^{B}\left(s^{\prime}, a^{*}\right)-Q^{A}(s, a)\right)\right. \\ Q^{B}(s, a) \leftarrow Q^{B}(s, a)+\alpha\left(r+\gamma\left(Q^{A}\left(s^{\prime}, a^{*}\right)-Q^{B}(s, a)\right)\right.\end{array}\right.$

Where $a^{*}=\operatorname{argmax}_{a} Q^{A}\left(s^{\prime}, a\right)$ to update the first Qfunction $Q^{A}, b^{*}=\operatorname{argmax}_{a} Q^{B}\left(s^{\prime}, a\right)$ updating $Q^{B}$. It should be noted that each update takes place using the value of the mutual Q-function for the next state $s^{\prime}$, eliminating overestimation bias.

Nonetheless, the original and different variants of the DQL algorithm, including Clipped-DQL [24] and Weighted-DQL [25] are still associated with high underestimation bias which is not desirable in many problems and may lead to unsatisfactory performance. A developed algorithm to deal with scheduling problems in an IoE-based smart grid is needed to address two critical concerns as follows.

\section{A. Step 1: Making a trade-off between underestimation and overestimation biases}

Inductive bias in a learning algorithm is a prior probability that shows the preference of an event before running the test. Fitting inductive bias with the nature of the problem is a crucial issue accomplishing a proper generalization performance. In simple words, overestimation and underestimation are not necessarily unfavorable in essence, and making a trade-off between these biases enhances the program's functioning. Overestimation bias leads to more exploration, while underestimation bias results in higher exploitation.

To address the above-mentioned concern selecting the estimators in a probabilistic manner for updating the value function is proposed. The algorithm takes advantage of two separated unbiased estimators $Q^{A}$ and $Q^{B}$ that can be selected for the updating rule by the probability of $\delta$ and $1-\delta$, respectively. As a hyperparameter, $\delta$ adjusts the positive and negative biases based on the problem environment.

As Figure 2 illustrates, a plain MDP model has three states (i.e., A, B, and C) inspired by user's consumption statuses, including normal, above normal, and less than normal. Each state has $\omega+1$ actions so that one action transitions to the next state with a deterministic reward $r=0$, and $\omega$ actions transition to the terminate state with a stochastic reward from states A, B, and C, which are clarified by Equations (48) to (50), respectively.

$r=\mu+U(-x,+x)=\mu+\zeta$
$r=-\mu+U(-x,+x)=-\mu+\zeta$
$r=\frac{\mu}{2}+U(-x,+x)=0.5 \mu+\zeta$

Where $\mu$ denotes the average reward and $U(-x,+x)$ is a uniform distribution used to determine $\zeta$. 


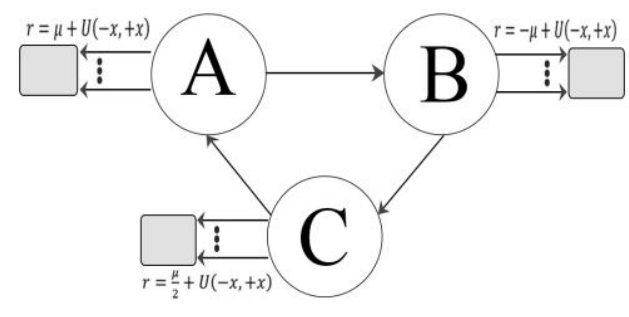

Figure 2. An example of episodic MDP with three non-terminal states

Suppose $\mu>0$, the stochastic regions from state A to termination and from state $\mathrm{B}$ to termination become high value and low value, respectively. In this condition, overestimation bias improves exploration in state A resulting in a better performance. Also, states $\mathrm{C}$ takes advantage of overestimation with a less slope. On the other hand, when $\mu<0$, underestimation bias helps regarding taking an action after state B and hurts others. This arrangement shows that eliminating overestimation and underestimation in many circumstances is not the case by itself, and making a trade-off based on the problem and environment is required.

\section{B. Step 2: Turning the tuned version into a Probably Approximately Correct (PAC) algorithm}

Simultaneous improvement in exploration and exploitation abilities leads to the high complexity of the model, especially sample complexity, which identifies the quantity of time and experience that the model requires to perform near optimal. Additionally, model-free algorithms (e.g., DDQL) lean on observed reward regardless of states transmissions resulting in higher sample complexity.

As a comprehensive RL-based solution, the proposed model needs to be developed as a PAC algorithm by applying a delay in the update rule achieving near-optimal performance while bounding the sample complexity. The designed algorithm restricts the sample complexity to be $O\left(S^{2} A\right)$ with $P[$ error $\leq \epsilon] \geq 1-\delta$. Where $S$ is state space, $A$ represents action space, $\delta$ denotes confidence parameter, and $\varepsilon$ is error parameter. The key point is that the estimator Q-value updates after $n$ attempts when Equation (51) is fulfilled [26].

$Q_{t}(s, a)-\left(\frac{1}{n} \sum_{i=1}^{n} r_{w_{i}}+\gamma \max _{a} Q_{w_{i}}\left(s_{w_{i}}, a\right)\right) \geq 2 \epsilon$

After the $n^{\text {th }}$ attempt the update takes place as shown in Equation (51) where $n$ denotes the number of attempts, $s_{w_{i}}$ and $r_{w_{i}}$ are $i^{\text {th }}$ recent next states and reward, respectively.

$Q_{t+1}(s, a)=\frac{1}{n} \sum_{i=1}^{n} r_{w_{i}}+\gamma \max _{a} Q_{w_{i}}\left(s_{w_{i}}, a\right)+\epsilon$

\section{Step 3: Forming P3DQL algorithm}

This research proposes the P3DQL algorithm, which is a combination of a new variant of DDQL and Delayed QLearning. First, the original version of DDQN is well-tuned, making a logical trade-off between positive and negative biases. Next, the algorithm developed into a PAC algorithm by applying a delay in update rule that bounds sample complexity. Although choosing the estimator for the updating rule in a probabilistic manner may cause higher problem complexity, developing the set of rules as a PAC algorithm guarantees superior learning efficiency.

As Algorithm 1 shows, the probabilistic DDQL takes advantage of two separated unbiased estimators. The update rules for both Q-functions (i.e., $Q^{A}$ and $Q^{B}$ ) adhere to update parameter $\beta$, where the probability of $\beta=1$ is $\delta$, likewise $\operatorname{Pr}(\beta=0)=1-\delta$. Then, the selection parameter $\psi$ defines whether $Q^{A}$ or $Q^{B}$ be updated. Hyperparameter $\delta$ adjusts positive and negative biases considering the problem environment so that the bigger $\delta$ results in higher underestimation. Contrariwise, lower $\delta$ is utilized when more exploration leads to better performance.

After initializing $Q^{A}$ and $Q^{B}$, two temporal buffer functions $U^{A}(s, a)$ and $U^{B}(s, a)$ store $n$ recent updates while the counter $l(s, a)$ defines the number of occurred updates. Each Q-function update is allowed only once Learning Flag $L F(s, a)$ is true. On the condition that no update takes place after a specific length of time, $\operatorname{LF}(s, a)$ turns into false.

The proposed method establishes a bias margin between underestimation and overestimation bounds. Both positive and negative biases are shrunk at the same time considering the following statements.

Lemma 1. Let $V=\left\{v_{1}, v_{2}, \ldots, v_{n}\right\}$ be a set of values and let $\alpha=$ $\min \left\{v_{1}, v_{2}, \ldots, v_{n}\right\}, \beta=\max \left\{v_{1}, v_{2}, \ldots, v_{n}\right\}$, and $v_{j}>v_{j+1}$. Then, $\alpha \leq \frac{1}{n} \sum_{i=1}^{n} v_{i} \leq \beta$ and $\alpha \leq \mathrm{E}\left\{V_{i}\right\} \leq \beta$.

Lemma 2. Let $V=\left\{v_{1}, v_{2}, \ldots, v_{n}\right\}$ be a set of values and let $\alpha=$ $\min \left\{v_{1}, v_{2}, \ldots, v_{n}\right\}, \beta=\max \left\{v_{1}, v_{2}, \ldots, v_{n}\right\}, Y=\{V\}-\alpha, W=$ $\{V\}-\beta$. Also, $\operatorname{Pr}\left(x_{i}{ }^{\Omega}\right)$ is the probability of $x \in\{\Omega \mid \Omega=$ $\{V, Y, W\}\}$. Then, $\operatorname{Pr}\left(x_{i}{ }^{\mathrm{Y}}\right) \max _{i}\left\{Y_{i}\right\}+\operatorname{Pr}\left(x_{i}{ }^{\mathrm{W}}\right) \max _{i}\left\{W_{i}\right\}<\beta$, and $\alpha<\operatorname{Pr}\left(x_{i}^{\mathrm{Y}}\right) \min _{i}\left\{Y_{i}\right\}+\operatorname{Pr}\left(x_{i}{ }^{\mathrm{W}}\right) \min _{i}\left\{W_{i}\right\}$.

These two lemmas are used to prove Theorem 1, which indicates the tuned version improves both negative and positive biases simultaneously. The theorem is as follows:

Theorem 1. Let $V=\left\{v_{1}, v_{2}, \ldots, v_{n}\right\}$ be a set of values, the expected value is maximized by $N$, and minimized by $M$, which are two subsets of $V$ (i.e., $N \subseteq V$, and $M \subseteq V$ ), and $N=$ $\left\{i \mid E\left\{V_{i}\right\}=\max _{j} V_{j}\right\}$, also $M=\left\{k \mid E\left\{V_{k}\right\}=\min _{j} V_{j}\right\}$. Let $T^{A}=$ $\left\{\tau_{1}^{A}, \tau_{2}^{A}, \ldots, \tau_{n}^{A}\right\}$ and $T^{B}=\left\{\tau_{1}^{B}, \tau_{2}^{B}, \ldots, \tau_{n}^{B}\right\}$ be two unbiased estimators so that $E\left\{V_{i}\right\}=E\left\{T^{A}{ }_{i}\right\}=\left\{T^{B}{ }_{i}\right\}$, also let $\bar{a}$ and $\underline{a}$ be two elements that maximize and minimize $T^{A}$. If $\theta_{u}$ and $\theta_{o}$ are lower and upper bound of bias in original DQN, then $\min _{i} E\left\{V_{i}\right\}=\theta_{u}<E\left\{T^{B}{ }_{\underline{a}}\right\}<E\left\{T^{B} \bar{a}\right\}<\theta_{o}=\max _{i} E\left\{V_{i}\right\}$

Proof. If $\underline{a} \in M$, and $\beta=1$, subsequently $E\left\{T^{B}{ }_{\underline{a}}\right\}=$ $\frac{1}{n} \sum_{i=1}^{n} \vartheta_{i}$, where $\theta_{u}=\vartheta_{1}=\min _{i} E\left\{V_{i}\right\}$, and $\vartheta_{j+1} \geq \vartheta_{j}$. Then, $\min _{i} E\left\{V_{i}\right\}=\theta_{u}<E\left\{T^{B}{ }_{a}\right\}$. If $\underline{a} \in N$, and $\beta=0$, then $\theta_{u}<$ $\vartheta_{1}$, and consequently $\min _{i} E\left\{V_{i}\right\}=\theta_{u} \ll E\left\{T^{B}{ }_{a}\right\}$. In the same way, if $\bar{a} \in N$, and $\beta=1$, accordingly $\left\{T^{B} \bar{a}\right\}=\frac{1}{n} \sum_{i=1}^{n} \vartheta_{i}$, where $\theta_{o}=\vartheta_{n}=\max _{i} E\left\{V_{i}\right\}$, and $\vartheta_{j} \geq \vartheta_{j-1}$. Afterward $E\left\{T^{B}{ }_{a}\right\}<\max _{i} E\left\{V_{i}\right\}=\theta_{o}$. If $\bar{a} \in N$, and $\beta=0$, then $\left\{T^{B} \bar{a}\right\}=\frac{1}{n} \sum_{i=1}^{n} \vartheta_{i}$, where $E\left\{T^{B}{ }_{a}\right\} \ll \theta_{o}=\max _{i} E\left\{V_{i}\right\}$. 


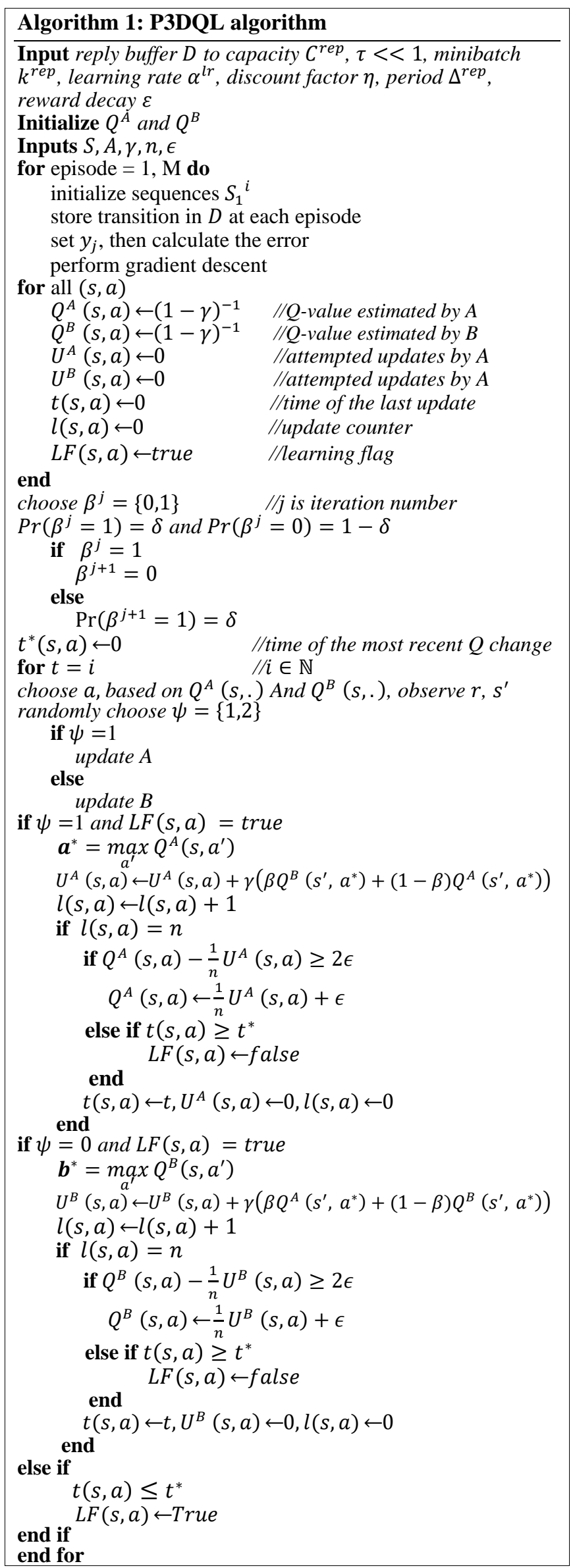

\section{Simulation Results}

The proposed P3DQL algorithm is evaluated using a large real-world dataset collected by Pecan Street [27], including PV generation and electricity consumption by every single device in different smart homes in Austin, TX. The information has been recorded for over three years every 15 minutes. Additionally, weather information and electricity retail prices are collected from the National Weather Service (NWS) [28] and Austin Energy [29], respectively. The simulation is tested using Python, 3.9.7 on a standard system with an Intel Core i7-97580H CPU with 16.0 GB of RAM.

\section{A. Multi-layer Grid Setup}

The model consists of 25 smart homes as NAs, where each NA includes major appliances, as mentioned in section 3 along with a $1 \mathrm{~kW}$ PV system and an EV. A group of five or six NAs forms an NH besides a set of ESS, SPV, and EVC. Consequently, five NHs are modeled where several NAs participate in multiple NHs. Similarly, two WANs are formed by different NHs. Table 1 clarifies the structure and components of different layers, where $i$ and $j$ indicates the ID of participated NAs, and NHs, respectively.

TABLE 1

THE STRUCTURE AND COMPONENTS OF NAS, NHS, AND WANS.

\begin{tabular}{|c|c|c|c|c|}
\hline Layer & Components & $\begin{array}{c}\text { ESS } \\
(\mathbf{k W})\end{array}$ & $\begin{array}{c}\text { SPV } \\
(\mathbf{k W})\end{array}$ & $\begin{array}{c}\text { EVC } \\
(\mathbf{k W})\end{array}$ \\
\hline All NAs & All major devices & - & - & - \\
\hline NH-1 & $i \in\{1 \rightarrow 5\}$ & 4 & 5.2 & 8 \\
\hline NH-2 & $i \in\{4 \rightarrow 9\}$ & 5 & 6.2 & 10 \\
\hline NH-3 & $i \in\{10 \rightarrow 14\} \cup\{1\}$ & 5 & 6.2 & 10 \\
\hline NH-4 & $i \in\{15 \rightarrow 20\}$ & 6 & 6.2 & 10 \\
\hline NH-5 & $i \in\{21 \rightarrow 25\} \cup\{9\}$ & 6 & 6.2 & 10 \\
\hline WAN-1 & $j \in\{1,2,3\}$ & 14 & 17.6 & 28 \\
\hline WAN-2 & $j \in\{2,4,5\}$ & 17 & 18.6 & 30 \\
\hline
\end{tabular}

All ESSs are Zinc bromide flow batteries with titanium electrodes, where $S O C_{E S S_{\min }}=1-2 \mathrm{~kW}$, and $S O C_{E S S_{\max }}=$ $12-20 \mathrm{~kW}$, and $\lambda_{\text {bat }}=0.75$. All ESSs are $5.2 \mathrm{~kW}$ solar kit Canadian 400 black with Enphase micro-inverter or $6.2 \mathrm{~kW}$ solar kit Q-Cells with Generac hybrid inverter. Finally, diesel generators supply EVCs, where quadratic coefficients of the generation cost function $\beta_{1}=0.001 \$ / k W^{2}, \quad \beta_{2}=$ $0.042 \$ / k W h$, and $\beta_{3}=0.4 \$ / h$. It should be noted that incentive factor $\Omega$ and penalty factor $\Phi$ are defined based on the historical consumption of each user. Also, loss coefficients and transmission fee coefficients among all units are set based on standard values in the real-world power system and electricity market.

\section{B. P3DQN Setup}

The prioritized experience replay technique is utilized to eliminate instability caused by the significant correlation between actions and states. The size of the replay buffer is $10^{6}$, while the number of training sets processed during each stochastic gradient descent update is $k^{r e p}=64$, which is called the minibatch size. Also, $\varepsilon$-greedy increment $\varepsilon=$ 
0.995 , decay step is 50 , the learning rate is $\alpha^{\mathrm{Ir}}=0.015$, and discount factor $\eta=0.9$.

It should be noted that a four-layer deep neural network is designed to simulate the model under given system parameters, historical consumption, retail price, weather data, and solar PV generation. The number of inputs and outputs are defined based on the total number of time slots during a day which is 96 , while the number of neurons is taken by trial and error to select the best fit number. An Adam optimizer is utilized with the defined learning rate $\alpha^{\text {lr }}$ The number of neurons in each layer is 1500 , and the activation function is ReLU. Ultimately, running the algorithm using different $\delta$ indicates that $\operatorname{Pr}(\beta=1)=0.65$ performs better than other combinations to solve this specific scheduling problem.

\section{Training Process}

Before investigating the effect of the algorithm on the load curve and cost in each grid layer, the training results are provided. Adapting the agents to the environment is a progressive procedure that is more fluctuating at the beginning steps due to lots of random choices obtaining a better exploration. Figure 3 shows the trend of reward during the training stage, where after 300 episodes, the agent learns to behave near the optimal policy. To simplify the explanation, the reward for a NA is demonstrated since other layers also follow the same trends. Although oscillation in the P3DQN is higher than other tested algorithms at the beginning but it achieves higher reward and demonstrates more stability in terms of learning and rewarding.

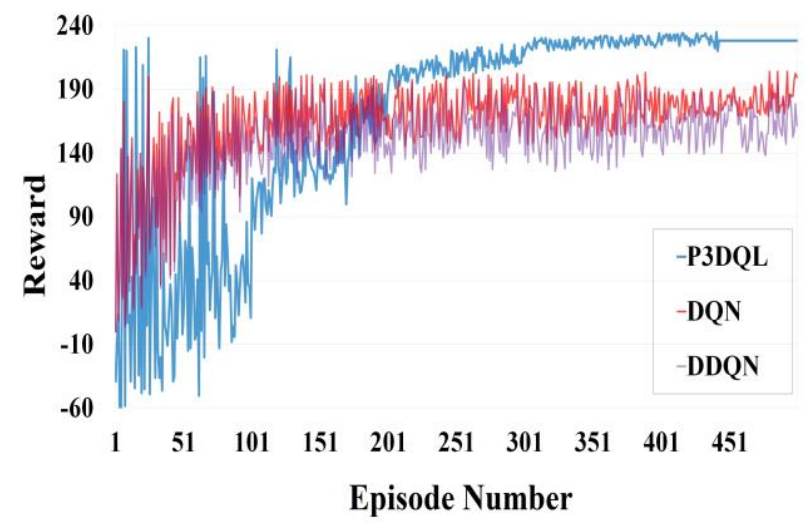

Figure 3. The rewards of three algorithms during training

Table 2 indicates while the overestimation of DQL has been reduced by DDQL, the proposed algorithm decreased the underestimation bias. All three algorithms were trained in different layers under exact conditions comparing average expected and actual returns after 10 random seeds.

TABLE 2

RESULT COMPARISON AMONG DIFFERENT METHODS

\begin{tabular}{|c|c|c|c|}
\hline Method & Expected return & Actual return & \% Error \\
\hline P3DQL & 178.7124 & 182.3781 & -0.0201 \\
\hline DDQL & 111.3924 & 145.4968 & -1.2344 \\
\hline DQL & 175.3237 & 136.3750 & +1.2856 \\
\hline
\end{tabular}

Additionally, after 436 episodes, the training stage is completed, and there will be no more updates by the estimators. The results show that the sample efficiency of the P3DQL has been enhanced by 39\% and 32\% comparing DQL and DDQL, respectively.

\section{Numerical Results: Load Profile}

The P3DQN algorithm is tested to discover the outcomes for different grid layers in terms of peak reduction and flatting the load curve. The trained algorithm is utilized to schedule the energy entities over four separated weeks in different seasons, taking weather and irradiance differences during a year into account, considering holidays and weekend effects besides the effect of seasonal appliances. Figure 4 shows the average load curve of major shiftable appliances, fixed loads, and power generated by the solar system in NA number 14 before applying the proposed scheduling algorithm.

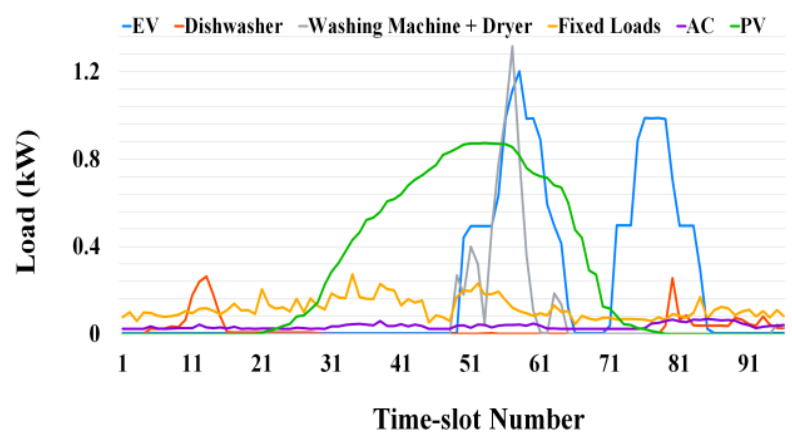

Figure 4. Load curve of different components in an NA

Tables 3 summarizes the results, where \%PAPR represents the percentage of reduction in Peak to Average Power Ratio (PAPR).

TABLE 3

LOAD PROFILE IMPROVEMENT WITH DIFFERENT ALGORITHMS

\begin{tabular}{|l|c|c|c|c|c|}
\hline \multirow{2}{*}{ Layer } & Metric & Initial & P3DQL & DDQL & DQL \\
\hline \multirow{2}{*}{$\begin{array}{l}\text { NA } \\
\# 14\end{array}$} & Peak $(\mathrm{kW})$ & 2.793 & 2.152 & 2.316 & 2.513 \\
\cline { 2 - 6 } & Variance & 0.805 & 0.695 & 0.741 & 0.785 \\
\cline { 2 - 6 } & $\%$ PAPR & - & $12.9 \%$ & $11.3 \%$ & $8.2 \%$ \\
\hline \multirow{2}{*}{$\begin{array}{l}\text { NH } \\
\# 4\end{array}$} & Peak $(\mathrm{kW})$ & 13.715 & 9.833 & 11.261 & 12.574 \\
\cline { 2 - 6 } & Variance & 2.895 & 2.515 & 2.621 & 2.752 \\
\cline { 2 - 6 } & $\%$ PAPR & - & $12.1 \%$ & $10.1 \%$ & $7.5 \%$ \\
\hline \multirow{2}{*}{$\begin{array}{l}* \\
\#\end{array} 2$} & Peak $(\mathrm{kW})$ & 33.451 & 23.740 & 29.451 & 32.745 \\
\cline { 2 - 6 } & Variance & 16.286 & 13.874 & 14.753 & 15.123 \\
\cline { 2 - 6 } & $\%$ PAPR & - & $11.8 \%$ & $9.8 \%$ & $6.9 \%$ \\
\hline
\end{tabular}

Utilizing P3DQN reduces peak load by $27.6 \%$ in July, $8.3 \%$ in October, $12.1 \%$ in February, and $13.9 \%$ in April. Then, the algorithm applies to the NH-4, while two different scenarios are examined, studying the effect of shared battery storage, shared PV, and EV charging station. In the first scenario with 6 NAs, the peak load decreases by $25.1 \%, 7.9 \%$, $11.4 \%$, and $13 \%$ in July, October, February, and April, respectively. The second scenario, including $6 \mathrm{NAs}$, a $6 \mathrm{~kW}$ ESS, a $6.2 \mathrm{~kW} \mathrm{SPV}$, and a $10 \mathrm{~kW}$ EVC shows outstanding 
performance in terms of peak reduction in July by $28.2 \%$ reduction. In the same way, the algorithm is tested on WAN-2, which is a combination of NH-2, NH-4, and NH-5.

\section{E. Numerical Results: Cost}

This section analyzes the performance of the P3DQL in terms of cost reduction taking three different tariff types into account, including flat tariff, Time of Use (ToU), and RealTime Pricing (RTP). Since the flat tariff scenario assumes that the electricity price does not change during the day, the only electricity scheduling feature is managing the generated power by the solar system. Consequently, the effect of utilizing any algorithm is slight. Alternatively, as indicated in Figure 5, both ToU and RTP demonstrate outstanding results, where the cost is deducted by $28.3 \%, 29.2 \%$, and $29.4 \%$ in NA-14, NH4 , and WAN-2, respectively.

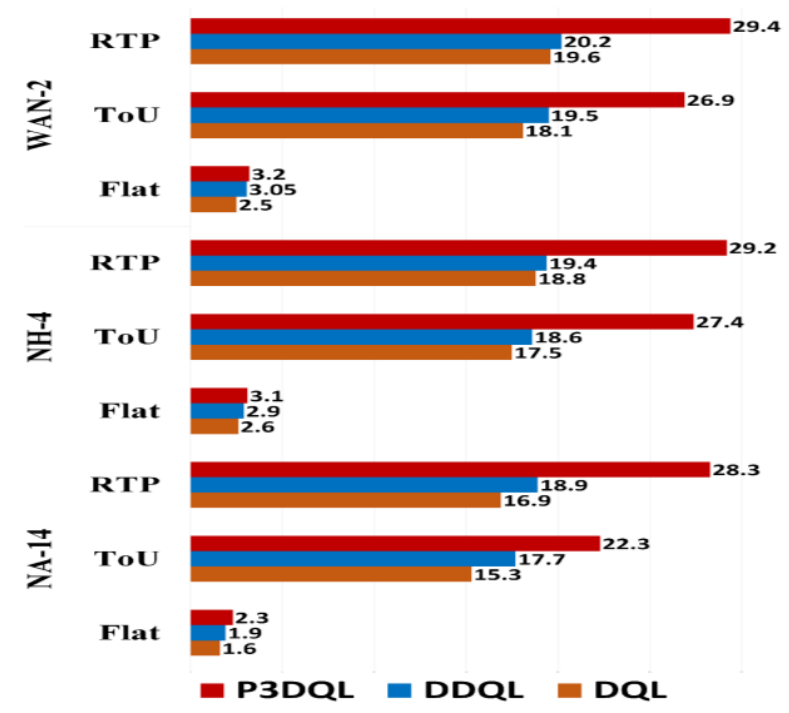

Figure 5. Different scenarios of cost reduction

\section{F. Results Comparison}

This section compares various methods, including DQLbased and DPG-based algorithms, and Long Short-Term Memory (LSTM) as modern methods, and one of the most used traditional methods for solving scheduling problems, which is Mixed Integer Linear Programming (MILP). Peak reduction and cost minimization are investigated as the two influential criteria. To ease of comparison, all results in Table 4 are presented, indicating the percentage of peak and cost reductions.

TABLE 4

RESULT COMPARISON AMONG DIFFERENT METHODS

\begin{tabular}{|c|c|c|}
\hline Method & Peak Reduction \% & Cost Reduction \% \\
\hline MILP [30] & 9.8 & N/A \\
\hline LSTM [31] & 10.4 & N/A \\
\hline DQL [19] & 12.1 & 20.01 \\
\hline DDPG [20] & 11.2 & 12.3 \\
\hline DPG [19] & 26.3 & 25.4 \\
\hline Proposed algorithm & 29.2 & 29.8 \\
\hline
\end{tabular}

\section{Conclusion}

This research proposed a novel model-free Q-Learning based algorithm for scheduling various types of energy components in a multi-layer IoE-enabled smart grid. Different layers are modeled with a highly dynamic environment, where participating in an $\mathrm{NH}$ or WAN may change based on user policy. Additionally, applying shared storage and energy producer units besides incentive-based pricing indicates the necessity of an intelligent scheduling algorithm.

Traditional scheduling methods are not capable of addressing this problem since comprehensive simulating of the environment is practically impossible due to different types of uncertainties and gigantic dimensionality of the problem. Moreover, DDQN and DPG based algorithms suffer from underestimation bias and discretization issues, respectively. Furthermore, stability and learning efficiency are two crucial metrics that need to be considered. Accordingly, the P3DQL algorithm has been proposed making an adjustable trade-off between positive and negative biases ensuring high efficiency besides lower complexity of the algorithm.

The proposed model has been tested on a large real-world dataset validating the algorithm's effectiveness regarding peak clipping, reducing PAPR, and cost reduction in different grid layers. Comparing the results with other applied methods on the same dataset in the literature indicated the superiority of the P3DQL by $28.2 \%$ peak clipping, $12.9 \%$ PAPR reduction, and $29.4 \%$ cost saving.

\section{REFERENCES}

F. Li, J. Qin, and W. X. Zheng, "Distributed Q-LearningBased Online Optimization Algorithm for Unit Commitment and Dispatch in Smart Grid," IEEE Transactions on Cybernetics, vol. 50, no. 9, pp. 41464156, Sep. 2020.

[2] H. M. Ruzbahani, A. Rahimnejad, and H. Karimipour, "Smart Households Demand Response Management with Micro Grid," 2019 IEEE Power and Energy Society Innovative Smart Grid Technologies Conference, ISGT 2019, Feb. 2019.

[3] H. M. Rouzbahani, H. Karimipour, and L. Lei, "A review on virtual power plant for energy management," Sustainable Energy Technologies and Assessments, vol. 47, p. 101370, Oct. 2021.

[4] J. Li, W. Shi, N. Zhang, and X. Shen, "Delay-Aware VNF Scheduling: A Reinforcement Learning Approach with Variable Action Set," IEEE Transactions on Cognitive Communications and Networking, vol. 7, no. 1, pp. 304-318, Mar. 2021.

[5] Y. Pan, "Heading toward Artificial Intelligence 2.0," Engineering, vol. 2, no. 4, pp. 409-413, Dec. 2016.

[6] D. Zhang, X. Han, and C. Deng, "Review on the research and practice of deep learning and reinforcement learning in smart grids," CSEE Journal of Power and Energy Systems, vol. 4, no. 3, pp. 362-370, Sep. 2018.

[7] Q. Zhang, K. Dehghanpour, Z. Wang, and Q. Huang, "A Learning-Based Power Management Method for Networked Microgrids under Incomplete Information," IEEE Transactions on Smart Grid, vol. 11, no. 2, pp. 1193-1204, Mar. 2020. 
[8] M. A. Lopes Silva, S. R. de Souza, M. J. Freitas Souza, and A. L. C. Bazzan, "A reinforcement learning-based multi-agent framework applied for solving routing and scheduling problems," Expert Systems with Applications, vol. 131, pp. 148-171, Oct. 2019.

[9] Y. Ji et al., "Data-Driven Online Energy Scheduling of a Microgrid Based on Deep Reinforcement Learning," Energies 2021, Vol. 14, Page 2120, vol. 14, no. 8, p. 2120, Apr. 2021.

[10] T. Remani, E. A. Jasmin, and T. P. I. Ahamed, "Residential Load Scheduling with Renewable Generation in the Smart Grid: A Reinforcement Learning Approach," IEEE Systems Journal, vol. 13, no. 3, pp. 3283-3294, Sep. 2019.

[11] S. Lee and D. H. Choi, "Reinforcement Learning-Based Energy Management of Smart Home with Rooftop Solar Photovoltaic System, Energy Storage System, and Home Appliances," Sensors 2019, Vol. 19, Page 3937, vol. 19, no. 18 , p. 3937 , Sep. 2019.

[12] M. Khan, J. Seo, and D. Kim, "Real-Time Scheduling of Operational Time for Smart Home Appliances Based on Reinforcement Learning," IEEE Access, vol. 8, pp. 116520-116534, 2020.

[13] W. Cui and W. Yu, "Scalable Deep Reinforcement Learning for Routing and Spectrum Access in Physical Layer," IEEE Transactions on Communications, vol. 69, no. 12 , pp. 8200-8213, Dec. 2021.

[14] S. Lee and Y. H. Lee, "Improving Emergency Department Efficiency by Patient Scheduling Using Deep Reinforcement Learning," Healthcare 2020, Vol. 8, Page 77, vol. 8, no. 2, p. 77, Mar. 2020.

[15] Y. Wang et al., "Multi-objective workflow scheduling with deep-Q-network-based multi-agent reinforcement learning," IEEE Access, vol. 7, pp. 39974-39982, 2019.

[16] D. Domínguez-Barbero, J. García-González, M. A. SanzBobi, and E. F. Sánchez-Úbeda, "Optimising a Microgrid System by Deep Reinforcement Learning Techniques," Energies 2020, Vol. 13, Page 2830, vol. 13, no. 11, p. 2830, Jun. 2020.

[17] X. Lu, X. Xiao, L. Xiao, C. Dai, M. Peng, and H. V. Poor, "Reinforcement Learning-Based Microgrid Energy Trading With a Reduced Power Plant Schedule," IEEE Internet of Things Journal, vol. 6, no. 6, pp. 1072810737, Dec. 2019.

[18] Z. Wan, H. L. H. He, and D. Prokhorov, "Model-Free Real-Time EV Charging Scheduling Based on Deep Reinforcement Learning," IEEE Transactions on Smart Grid, 2018.

[19] E. Mocanu et al., "On-Line Building Energy Optimization Using Deep Reinforcement Learning," IEEE Transactions on Smart Grid, vol. 10, no. 4, pp. 3698-3708, Jul. 2019.

[20] H. M. Chung, S. Maharjan, Y. Zhang, and F. Eliassen, "Distributed Deep Reinforcement Learning for Intelligent Load Scheduling in Residential Smart Grids," IEEE Transactions on Industrial Informatics, vol. 17, no. 4, pp. 2752-2763, Apr. 2021.

[21] A. Lassetter and E. Cotilla-Sanchez, "Exponential modeling of equipment degradation in the grid for more reliable contingency analysis," SEST 2021 - 4th International Conference on Smart Energy Systems and Technologies, Sep. 2021.
[22] D. M. Minhas and G. Frey, "Modeling and Optimizing Energy Supply and Demand in Home Area Power Network (HAPN)," IEEE Access, vol. 8, pp. 2052-2072, 2020.

[23] H. van Hasselt, A. Guez, and D. Silver, "Deep Reinforcement Learning with Double Q-learning," 30th AAAI Conference on Artificial Intelligence, AAAI 2016, pp. 2094-2100, Sep. 2015, Accessed: Jan. 19, 2022.

[24] S. Fujimoto, H. van Hoof, and D. Meger, "Addressing Function Approximation Error in Actor-Critic Methods," 35th International Conference on Machine Learning, ICML 2018, vol. 4, pp. 2587-2601, Feb. 2018, Accessed: Jan. 20, 2022.

[25] Z. Zhang, Z. Pan, and M. J. Kochenderfer, "Weighted double Q-learning," IJCAI International Joint Conference on Artificial Intelligence, vol. 0, pp. 34553461, 2017.

[26] A. L. Strehl, L. Lihong, E. Wiewiora, J. Langford, and M. L. Littman, "PAC model-free reinforcement learning," ACM International Conference Proceeding Series, vol. 148, pp. 881-888, 2006.

[27] L. Certification, "Pecan Street Dataport," 2020. https://www.pecanstreet.org/dataport/

[28] N. N. W. S. US Department of Commerce, "National Weather Service".

[29] "Residential Electric Rates \& Line Items." https://austinenergy.com/ae/rates/residentialrates/residential-electric-rates-and-line-items.

[30] T. Buechler, F. Pagel, T. Petitjean, M. Draz, and S. Albayrak, "Optimal Energy Supply Scheduling for a Single Household: Integrating Machine Learning for Power Forecasting," Proceedings of 2019 IEEE PES Innovative Smart Grid Technologies Europe, ISGTEurope 2019, Sep. 2019.

[31] J. T. Meyer, L. A. Agrofoglio, J. Clement, Q. Liu, O. Yurdakul, and S. Albayrak, "Multi-objective residential electricity scheduling based on forecasting generation and demand via LSTM," IEEE PES Innovative Smart Grid Technologies Conference Europe, vol. 2020October, pp. 270-274, Oct. 202. 